\title{
Extended Service Integration: Towards "Manufacturing" SLA
}

\author{
Frédérique Biennier, Loubna Ali and Anne Legait \\ INSA LYON, LIESP. INSA de Lyon, Bat B. Pascal, 69621 \\ Villeurbanne Cédex - FRANCE \\ \{frederique.biennier, loubna.ali, anne.legait\}@insa-lyon.fr, \\ WWW home page: http://liesp.insa-lyon.fr
}

\begin{abstract}
Market constant evolution towards more and more customisation and call for "product/service" increases to need of agile and lean organisations, making an heavy use of information and communication technologies. To bring the necessary openness, interoperability and agility features to the enterprises information systems, one can use fruitfully Service Oriented Architecture. Already used at a business level as a potential interoperable and integrating framework, this technology must be adapted to define manufacturing services and to take into account manufacturing constraints, namely time constraint and security integration. In this paper, we propose a global framework to define a "manufacturing service bus", paying a particular attention to the manufacturing service definition. We also show how mobile agents can be used to set dynamically monitoring systems.
\end{abstract}

\section{Keywords}

SOA, Interoperability, SLA, manufacturing agreements, ESB

\section{Context}

Due to the economical context involving more and more customisation and "service oriented products", enterprises have to adapt their organisational strategy: while focusing on their core business, outsourcing or collaborative strategies must be set to fit the market requirements (i.e. getting a critical size and being able to provide a high service level to the consumer). These organisational trends rely on an heavy use of information and communication technologies calling for agility, i.e. the ability to answer to structural changes quickly (client requests, technological or activity changes, supplier management...), for reducing waste (leading to lean manufacturing organisation) and for developing a product/service strategy. This involves 
reorganising the enterprise according to the services it can propose and defining "manufacturing services", taking into account industrial constraints (security, time constraints, quality, management strategy consistency...).

To fit the openness and required agility levels involved by the enterprise constant changes according to the market evolution, one can reorganise the information System (IS) according to a Service Oriented Architecture (SOA). SOA organises a component based architecture, namely services, that are mostly business process oriented: thanks to the orchestration level, elementary services are composed according to the business process specification and orchestrated. As services provide well identified interfaces (mostly defined in XML format), they can be fruitfully used to bring the necessary interoperability level whereas the composition and orchestration mechanisms support the IS agility (figure 1).

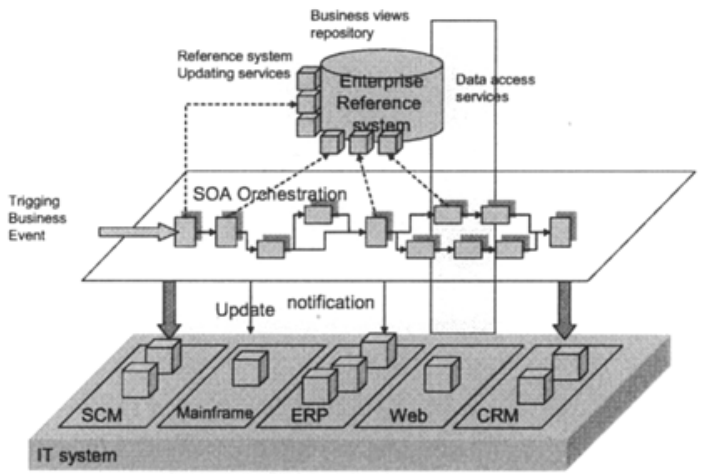

Figure 1: Interconnection of information system thanks to SOA from (Rivard et al. 2005), pp.25 and 39

Implementing a SOA requires both a middleware technology to enable service integration AND a distributed communication services. Focusing on the middleware side, SOA requires first to define service repository, a business service choregrapher (in charge of composition and orchestration) as well as a middleware engine in charge of routing, message transformation, security control... Such functions are commonly implemented in enterprise service bus which provides access to external services (Schmidt et al. 2004) (figure 2).

After the composition process, orchestrating these different services leads to a "service chain" organisation" which may involves different partners. This involves defining contracts for the shared process and interface definition. Service underlying philosophy is that the execution should be environment independent. Such a requirement can not fit the manufacturing requirements where processes are heavily related to the exact hardware resource. Moreover, answering times and other Quality of Service parameters are not taken into account in the service contract. To support the "end to end" contractual relationships including QoS, Service Level Agreements (SLA) are set between "connected services", linking the different partners among the service chain. (figure 3). The required Quality of Service defined in SLA is described thanks to Key Performance Indicators and Key Quality Indicators, taken from patterns associated to the business and network services. 


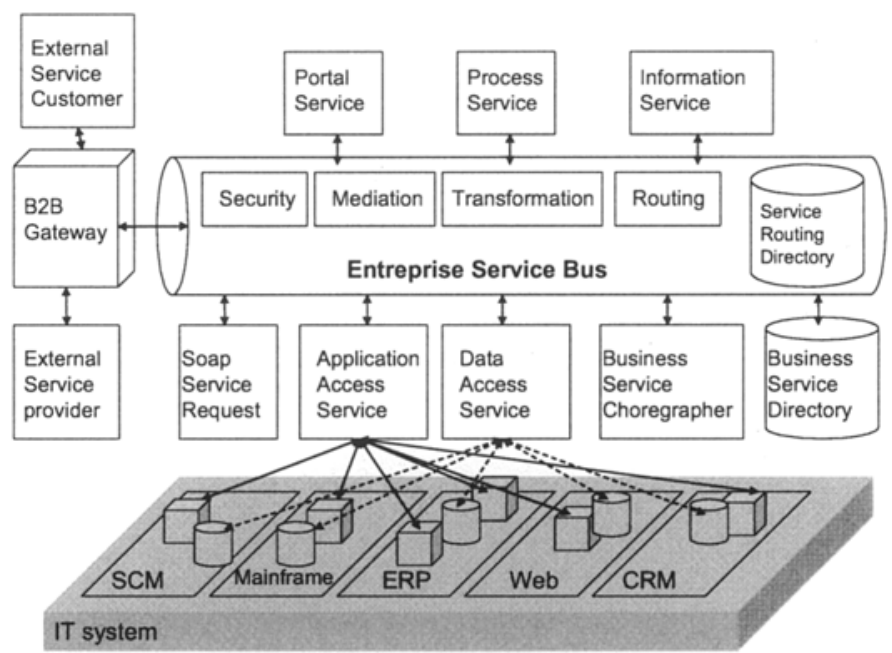

Figure 2: ESB position

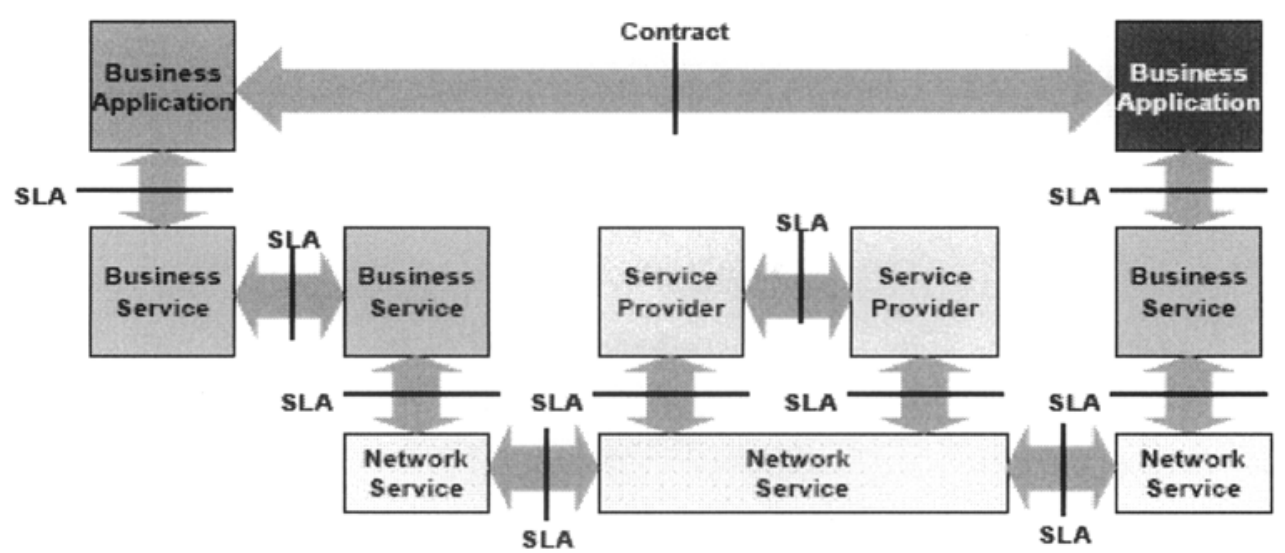

Figure 3: SLA and service combination (taken from (Open group, 2004), p.3)

Despite of the openness provided by the multi-level organisation, such a SLA framework is limited to the ICT infrastructure and does not fit an extended service chain organisation. In order to integrate a product-service dimension, we must first define how service-chain eco-system can be model to derive extended service models and then integrate manufacturing and management constraints in the SLA organisation. 


\section{Holistic manufacturing chain organisation}

To integrate manufacturing constraints, we propose to extend the "service chain" specification by adding a "manufacturing chain" based on a SCOR model (Supply Chain Council, 2000) in order to capture the management properties of the linked objects and to define the information to exchange. Due the plethoric variety of information which can be collected to describe and evaluate a manufacturing chain, we propose to establish a multi-dimensional modelling framework which can be enriched incrementally. We define a manufacturing chain recursively either as a manufacturing chain which links a set of manufacturing services, or as a simple stand-alone service. Services can be described as a set of competencies (i.e. what the service can achieve) whereas their position in the manufacturing chain is defined as a role (i.e. a set of competencies according to which the service is selected and plays a part). Based on this definition, we propose to analyze and model the manufacturing services according to an incremental holistic model (gathering IT, industrial, legal, economical, human related... dimensions) associated to four different points of view:

1. The "Internal" Service View describes the service competencies (i.e. what the service can be used for), the service's own organization (i.e. how the service is designed to achieve the works it can do) and the service management agents (including set of indicators/dashboards, acquisition and building processes). The service's own organization and the service management sub-views are conditioned by the service type (i.e. pure human service, semi-IT supported (i.e. human service using allied IT related services) or IT supported (which may include a human part heavily connected to the IT support). To support incremental description as well as to include best practice capitalization, the service model is split among different levels associated to different purposes (security requirements, service competencies, interconnection rules, exchange constraints...).

2. The Service Chain View is related to the role a service plays in the chain This view represents the public part of the service model and uses the service interface (i.e. the service competencies) to select the convenient service to set an adapted service chain. This model gathers 2 dual views: the External view which describes the service chain competencies and the way it can be integrated in a higher level chain and the Internal view. This internal view is based on the composition process description, i.e. definition of a strategic assembly policy (i.e. description of the different roles and of the criteria used to select the convenient services) and "assembly controllers" used to conveniently propagate the different constraints after selecting the services and assembling them in a service chain. Of course, after achieving a composition process, the current service chain described as a "service workflow" is stored in the model. To support management and evaluation services, this internal service-chain submodel is also related to service chain management agents, used to evaluate the added-value creation / propagation among the service chain and to evaluate the chain component satisfaction, describing the alignment of each service goal within the service chain's global goal. 
3. Environmental View is used to describe the service chain selection policy (namely, goals and service chain roles, management criteria, strategic selection criteria as partner trust chain, localisation...). This view is related to the "exposition" of the service chain external view. Nevertheless, the environmental model is not reduced to a simple service chain exposition; it also includes the environmental impact of the service chain (mostly structuring impact). Then, management agents are used to orchestrate the service chain and service management sub-models: these environmental agents are used to select the convenient dashboard patterns, collect environmental information used to define composition criteria and aggregate performance indicators. According to a midterm exploitation of the collected data, this management system will be used to capitalize emerging behaviour to guide further composition selection criteria.

Gathering these different views leads to a multi-interface extended service model:

- "Conceptual service": this interface is used to the service "semantic, i.e. the service "competencies" (what is achieved by this service)

- Manufacturing interface: this interface describes HOW the manufacturing service will be achieved. It includes

o Product view: this interface gathers information on material and products (bill of material, management policy, quality indicator...) as well as on the production management strategy.

- Process view: this includes both the routing specification, potential resource constraints (this interface is used to couple the manufacturing service to the resources), process qualification according to the CMMI classification (Williams and Wegerson 2002). For this last point we use the information stored in the different parts of the information system to asset the process maturity level (i.e. the manufacturing process can be described; its results are evaluated in a dashboard, documented...).

SLA control interface: Real-time constraints are used to describe global QoS constraints to be included in SLAs whereas safety constraints are used to define pre-emptive or not pre-emptive services. This interface is coupled to the dynamic monitoring system to define dynamically the adapted QoS monitoring features

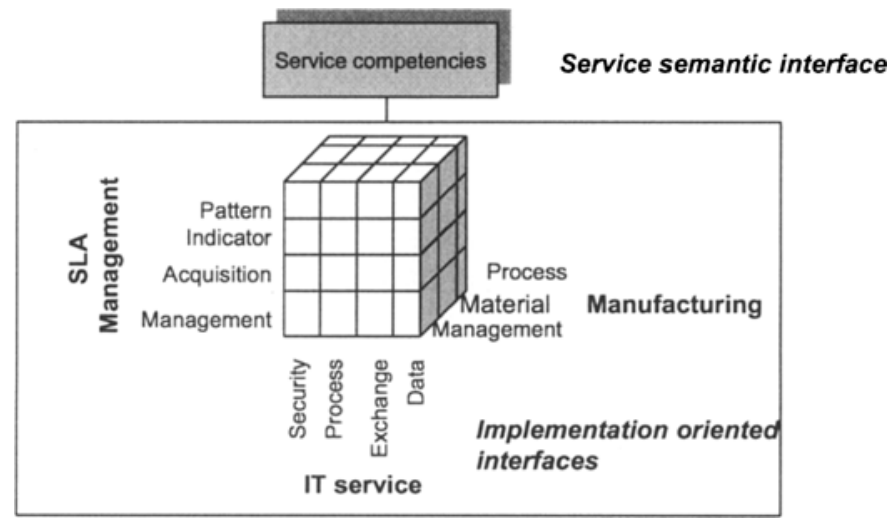

Figure 4: Extended service model 
IT View : this interface describes the connection with the IT support services / systems and addresses mostly information transformation (thanks to standards as B2MML or FDML), security services

\section{Towards a Manufacturing service bus}

To fit such an extended service, the underlying middleware organisation must be adapted in order to support information transformation (from the business to the manufacturing level), QoS and safety requirements. This leads to a manufacturing service bus (MSB) organisation able to process functional (service semantic, management strategy...) and non functional (namely security and QoS) interfaces. The MSB is based on the enterprise service bus organisation but, as it must integrate strong manufacturing constraints (i.e. service execution is related to the resources it is "run" on, QoS constraints, safety constraints leading to "pre-emptive" services...), the MSB integrates the manufacturing service composition system and the service orchestrator as well as a dynamic monitoring system:

- The service composition process enriches the service traditional semantic selection: our multi-criteria service selection uses information from the different interfaces: first services are selected "semantically" according the "competency oriented interface" and the implementation constraints are processes in the following order: first manufacturing constraints are processed (i.e. management strategy, process maturity level...) in order to define consistent manufacturing chains and then QoS constraints are taken into account by comparing the current context to the required performance level. IT service interface is not used to select or reject a manufacturing service: this view is lastly used to select the convenient IT services (information transformation, security components) to add in the composed service chain.

- Safety requirements (i.e. pre-empting resources in emergency cases) is implemented by adding execution priorities (standard or emergency) to the services while they are processed by the orchestrator and by defining" rollback" processes associated to the different service in order to get back after an emergency stop.

- Service mediation and transformation includes is based on B2MML (Emerson 2006) to implement information interface services towards business service providers and FDML to implement interfaces towards manufacturing resources

- Real-time constraints are taken into account by adding a QoS monitoring system.

This QoS monitoring system is implemented in a distributed way thanks to mobile agents. Based on the Aglet platform (Lange and Oshima 1998), we develop a set of different agents built incrementally to monitor the service chain (figure 6). The mobile agent architecture we use to implement the distributed management system consists in different agents types that can be assembled to set new management functions so that a dynamic management system is set. Three main types of agents are used: 
- Itinerary agents are in charge of the "mobility management", i.e. visit the different nodes belonging to a given itinerary to collect, configure... information before going back to the management node which has created them.

- Code agents are in charge of running the indicator building programs to set more complex indicators. They can be used in a distributed way in conjunction with itinerary agents.

- Action agents are used to orchestrate the area management, setting the convenient itinerary and action agents. These agents are organised into severa] categories(security management, QoS management, service management...).
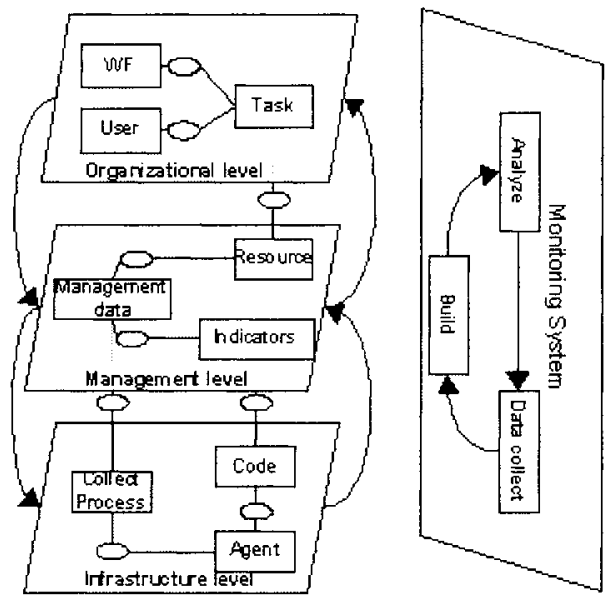

Figure 7: Monitoring system organisation

The management system is organised hierarchically and consists in three interacting monitoring algorithms running on the different nodes:

- The server node is in charge of information analysis and of notifying contextual information to the manager nodes. It manages the monitoring area information base, computes indicators by starting convenient code agents after receiving raw information via itinerary agents, extracts and sends back monitoring information. It can also launch an information acquisition process according to the information call received from manager nodes.

- The manager node is in charge of context analysis (either by analysing the information sent by the information server or by processing the notification sent by another area manager), identifies the convenient actions to achieve and set the itinerary agent (it define the visited node sequence and the convenient action to be launched on each node).

- Managed nodes algorithm is simpler: these nodes only wait for itinerary agents, and then identify the action part. Then, it has to get and install the code agent associated to the management action and set properly parameters. Lastly, at the end of the code agent process, a simple itinerary agent is started to send back the action result to the manager node. 


\section{Conclusion and further work}

To fit collaborative manufacturing environment, we proposed to adapt the business oriented service architecture to the manufacturing context. This leads us to define extended service, including different interfaces, as well as a "manufacturing service bus". This new middleware concept is based on the enterprise service bus but it also includes a manufacturing service composition process as well as a dynamic QoS monitoring system to fit real time constraints. We focus first on the real-time constraints, experiment various kinds of agents to collect information and set convenient configuration as well as to build more complex indicator. Nevertheless, we do not integrate by now the semantic associated to the different SCOR objects.

Acknowledgements: this work is supported by the INSA BQR project "Enterprise urbanism" and Rhone Alpes area council through the GOSPI Cluster project INTERPROD.

\section{References}

Lange D.B. and Oshima M., 1998. Programming and deploying java mobile agents with aglets, Addison-Wesley

Mathieu H., Ali L., Biennier F., 2006. A distributed management system: towards pro-active information system management in virtual enterprise. INCOM'06 Proceedings.

Open group, 2004. SLA Management handbook volume 4: Enterprise perspective, available at http://www.opengroup.org/pubs/catalog/g045.htm

Rivard F., Brendel C., Buche N., Delayre S., Mocaërr A., Nevers J., 2005. SOA et urbanisme: le rôle des architectures orientées service dans l'alignement des systèèmes d'information. Unilog White paper, available at http://admin.kermeet.com/Data/kmreed_informatique/event/F_374d0882e6f2c44d80bb816 b5c87600d42ba63161965c.pdf, 109p.

Schmidt M.T., Hutchinson B., Lambros P., Phippen R., 2005. The enterprise service bus. : Making service oriented architecture real. IBM System Journals, vol. 44, $n^{\circ} 4$, pp.781-797

Supply Chain Council, 2000. Supply Chain operations reference Model - SCOR Version 5.0, 255 pages

Williams R., Wegerson P., 2002. MINI CMMI(SM) (SE/SW/IPPD/SS Ver 1.1) Staged Representation. Cooliemon 